\title{
An intronic RNA structure modulates expression of the mRNA biogenesis factor Sus1
}

\author{
ALI ABUQATTAM, ${ }^{1,2}$ JOSÉ GALLGO, ${ }^{2}$ and SUSANA RODRÍGUEZ-NAVARRO ${ }^{1}$ \\ ${ }^{1}$ Gene Expression and RNA Metabolism Laboratory, Centro de Investigación Príncipe Felipe, Valencia 46012, Spain \\ ${ }^{2}$ Facultad de Medicina, Universidad Católica de Valencia, Valencia 46001, Spain
}

\begin{abstract}
Sus1 is a conserved protein involved in chromatin remodeling and mRNA biogenesis. Unlike most yeast genes, the SUS1 premRNA of Saccharomyces cerevisiae contains two introns and is alternatively spliced, retaining one or both introns in response to changes in environmental conditions. SUS1 splicing may allow the cell to control Sus1 expression, but the mechanisms that regulate this process remain unknown. Using in silico analyses together with NMR spectroscopy, gel electrophoresis, and UV thermal denaturation experiments, we show that the downstream intron (I2) of SUS1 forms a weakly stable, 37-nucleotide stem-loop structure containing the branch site near its apical loop and the $3^{\prime}$ splice site after the stem terminus. A cellular assay revealed that two of four mutants containing altered 12 structures had significantly impaired SUS1 expression. Semiquantitative RT-PCR experiments indicated that all mutants accumulated unspliced SUS1 pre-mRNA and/or induced distorted levels of fully spliced mRNA relative to wild type. Concomitantly, Sus1 cellular functions in histone $\mathrm{H} 2 \mathrm{~B}$ deubiquitination and mRNA export were affected in 12 hairpin mutants that inhibited splicing. This work demonstrates that 12 structure is relevant for SUS1 expression, and that this effect is likely exerted through modulation of splicing.
\end{abstract}

Keywords: Sus1; gene expression; splicing; yeast; NMR; RNA; structure; thermal stability

\section{INTRODUCTION}

Introns are removed from pre-mRNAs to create a mature transcript by the action of the splicing machinery (Wahl et al. 2009; Fica et al. 2013). Recent reports have shown that splicing contributes to the control of gene expression (Acuña and Kornblihtt 2014; Moehle et al. 2014) and can "fine-tune" different stages of the gene expression process by regulating the expression levels of key pathway components involved in RNP and ribosome biogenesis, transcription, and RNA transport (Johnson and Vilardell 2012). Some of these studies have focused on Saccharomyces cerevisiae Sus1, a factor involved in transcription and mRNA export (RodríguezNavarro et al. 2004; Galan and Rodríguez-Navarro 2012). Sus1 is part of the SAGA (Spt-Ada-Gcn5-Acetyltransferase) complex where it participates as a modulator of the deubiquitinase activity of Ubp8 (Köhler et al. 2006). It also interacts at the nuclear pore with TREX-2 (transcription export complex 2) and participates in mRNA export and genome stability (González-Aguilera et al. 2008). In addition, Sus1 transiently interacts during transcription elongation with RNA polymerase II and the export factors Yral and Mex67 (Pascual-García et al. 2008). An interesting feature of the SUS1 gene-highly

Corresponding authors: srodriguez@cipf.es, jose.gallego@ucv.es Article published online ahead of print. Article and publication date are at http://www.rnajournal.org/cgi/doi/10.1261/rna.054049.115. Freely available online through the RNA Open Access option. infrequent in the $S$. cerevisiae genome-is the fact that it contains two introns (Rodríguez-Navarro et al. 2004). The presence of two introns in the SUS1 gene opens up the possibility of a feedback mechanism involving splicing. A similar situation was found in the YRA1 gene encoding another essential mRNA export factor, whose expression was found to be controlled by different mRNA biogenesis steps (RodríguezNavarro et al. 2002; Preker and Guthrie 2006; Dong et al. 2007).

The functional relevance of the presence of two introns in the SUS1 pre-mRNA has been studied previously (Cuenca-Bono et al. 2011; Hossain et al. 2011). While intron 2 (I2) has consensus splice sequences, intron 1 (I1) is characterized by the presence of nonconsensus $5^{\prime}$ splice site (SS) (GUAUGA instead of canonical GUAUGU) and branch site (BS) (UACUGAC instead of UACUAAC). These sequences influence $\bar{S} U S 1$ splicing and as a consequence intron 1 is retained in $>15 \%$ of the SUS1 transcripts. Moreover, retention of I1 is affected by growth conditions, suggesting a potential role for splicing in regulating Sus 1 cellular function (Johnson and Vilardell 2012).

RNA structure is emerging as an important element in the regulation of gene expression, via modulation of interactions

(C) 2015 AbuQattam et al. This article, published in $R N A$, is available under a Creative Commons License (Attribution 4.0 International), as described at http://creativecommons.org/licenses/by/4.0/. 
with proteins and microRNAs or by arranging the spatial distribution of important sequences (Wan et al. 2015). For example, in the case of splicing intron RNA folding has been shown to influence $3^{\prime}$ SS selection through the masking of AG sequences inside double-helical stems, or by bringing BS and 3'SS sequences into functional proximity (Deshler and Rossi 1991; Rogic et al. 2008; Warf and Berglund 2010; Meyer et al. 2011; Pérez-Valle and Vilardell 2012). In S. cerevisiae, intronic RNA structures have been reported to play a role in autoregulating RPL30 and YRA1 expression at different levels (Dong et al. 2010; Meyer et al. 2011). Gaining insight into the role of RNA structure in controlling mRNA biogenesis at different stages is a major challenge in the field. In this article, we explore the regulatory role of a stem-loop structure formed by I2 of SUS1.

\section{RESULTS}

\section{Intron 2 of SUS1 forms a weakly stable hairpin structure in solution containing BS nucleotides in its apical loop}

The RNA-folding algorithm Mfold (Zuker 2003) predicted that I2 of S. cerevisiae SUS1 formed a 37-nucleotide (nt) stem-loop structure with BS nucleotides lying in the apical loop and $3^{\prime}$ SS nucleotides immediately after the $3^{\prime}$ stem terminus (Fig. 1; Hossain et al. 2011). The pre-mRNAs of several yeast species, selected from our previous evolutionary analysis of SUS1 (Cuenca-Bono et al. 2011), were also subjected to secondary structure predictions. The analyses revealed comparable putative hairpins formed by the second intron of S. pastorianus, K. thermotolerans, S. kudriavzevii, and S. mikatae. In these hairpins, the BS nucleotides similarly localized close to the apical loop, and the $3^{\prime}$ SS near the $3^{\prime}$ terminus of the base-paired stem (Fig. 1). These results were generally consistent with previous predictions in silico (Hossain et al. 2011).

To confirm the computational predictions, we next examined by NMR spectroscopy and gel electrophoresis the conformation of $S$. cerevisiae I2, represented by a $37-n$ t oligomer (hereafter named I2s) encompassing the hairpin predicted to be formed by I2 nucleotides 31-67 (Fig. 2). The electrophoretic results indicated the formation of a predominantly monomeric structure in different solution conditions. The NMR data revealed that I2s adopted the hairpin structure depicted in Figure 2. The presence of the $\mathrm{A}_{32} \mathrm{AAAGAGAA}_{40}$ : $\mathrm{U}_{58} \mathrm{UUUUU}$ UUUU $\mathrm{U}_{66}$ double-helical tract at the base of the hairpin was demonstrated by NOE interactions involving exchangeable and nonexchangeable resonances. The two G36: U62 and G38:U60 wobble pairs were clearly established by the characteristic chemical shift and NOE pattern of the G $\mathrm{H} 1$ and $\mathrm{U} \mathrm{H} 3$ iminos (Fig. 2A; Supplemental Fig. S1A, crosspeaks a and b). Likewise, diagnostic A $\mathrm{H} 2-\mathrm{U} \mathrm{H} 3$ interactions allowed identification of eight Watson-Crick A:U pairs in the I2s hairpin (Fig. 2A; Supplemental Fig. S1B). A35:U63, A37:U61, and A39:U59 were assigned from NOE contacts involving protons of G36:U62 and G38:U60 (Supplemental Fig. S1A, crosspeaks c-g, i, and j; Supplemental Fig. S1C, crosspeaks $\mathrm{k}, \mathrm{l}, \mathrm{n}$, and q). The remaining four A:U pairs of the lower stem were identified by cross-strand A H2- $\mathrm{U}$ H3 NOEs (indicated by solid horizontal arrows in Supplemental Fig. S1B), as well as by sequential intra-strand and cross-strand $\mathrm{H} 2-\mathrm{H} 1^{\prime}$ NOEs typical of canonical RNA stems (Supplemental Fig. S1C).

The NMR data were also consistent with the presence of the two Watson-Crick pairs forming the $\mathrm{A}_{45} \mathrm{C}_{46}: \mathrm{G}_{52} \mathrm{U}_{53}$ upper stem below the apical loop. The A45:U53 pair was suggested by the remaining A $\mathrm{H} 2-\mathrm{U} \mathrm{H} 3$ crosspeak, whose resonances were not connected by NOE interactions with the remaining A:U pairs of the system (Supplemental Fig. S1B,C). Diagnostic crosspeaks between guanine imino and cytosine amino protons that were unrelated to the terminal C67 residue allowed identification of the C46:G52 pair (Supplemental Fig. S1A, crosspeak h). There were additional imino resonances in the I2s spectrum (Fig. 2A) whose chemical shifts were consistent with the formation of G:U pairs in the CGUU:GGAA internal loop separating the two canonical stems, but they exchanged too quickly with the solvent to be assigned.

The stability of the hairpin formed by intron 2 of SUS1 was evaluated with UV-thermal denaturation experiments. The results indicated that this stem-loop structure had low thermal stability: the I2s hairpin melted at $26^{\circ} \mathrm{C}$ in the absence of $\mathrm{NaCl}$ and at $36^{\circ} \mathrm{C}$ with $100 \mathrm{mM} \mathrm{NaCl}$, in ionic conditions approximately similar to those found in a cellular environment. The thermal denaturation curves were reversible in all cases (Fig. 2C; Supplemental Fig. S2).

\section{Partial deletion of the stem-loop structure formed by intron 2 modulates $S$. cerevisiae SUS1 splicing}

The presence of a hairpin structure formed by I2 of SUS1 in $S$. cerevisiae together with its conservation in several yeast species suggested a possible functional relevance. To address this question, we studied an I2 deletion mutant (I2-mut1) in which the double-helical stem of the predicted hairpin was shortened by 9 base pairs (bp) while the BS was maintained in the apical loop and the $3^{\prime}$ SS after the stem (Fig. 3A). This mutant had $18 \mathrm{nt}$ less relative to wild-type I2, but all intronic features relevant for splicing were well within functional range: the total size of the mutant intron was $52 \mathrm{nt}$ (the minimum size has been proposed to be $50 \mathrm{nt}$ ), the distance between the 5'SS and the BS was $28 \mathrm{nt}$ (greater than the required $25 \mathrm{nt}$ minimum), and there were $12 \mathrm{nt}$ between the BS and the $3^{\prime}$ SS (10 nt are necessary in this case as a minimum) (Meyer et al. 2011; Pérez-Valle and Vilardell 2012). To evaluate the expression efficiency of this mutant in vivo, we used the CUP1 reporter system (Lesser and Guthrie 1993). cup $1 \Delta$ cells were transformed with one of the following reporter constructs: pACT1-CUP1, pSUS1g-CUP1 (containing a SUS1 gene with wild-type I1 and I2 introns), and 

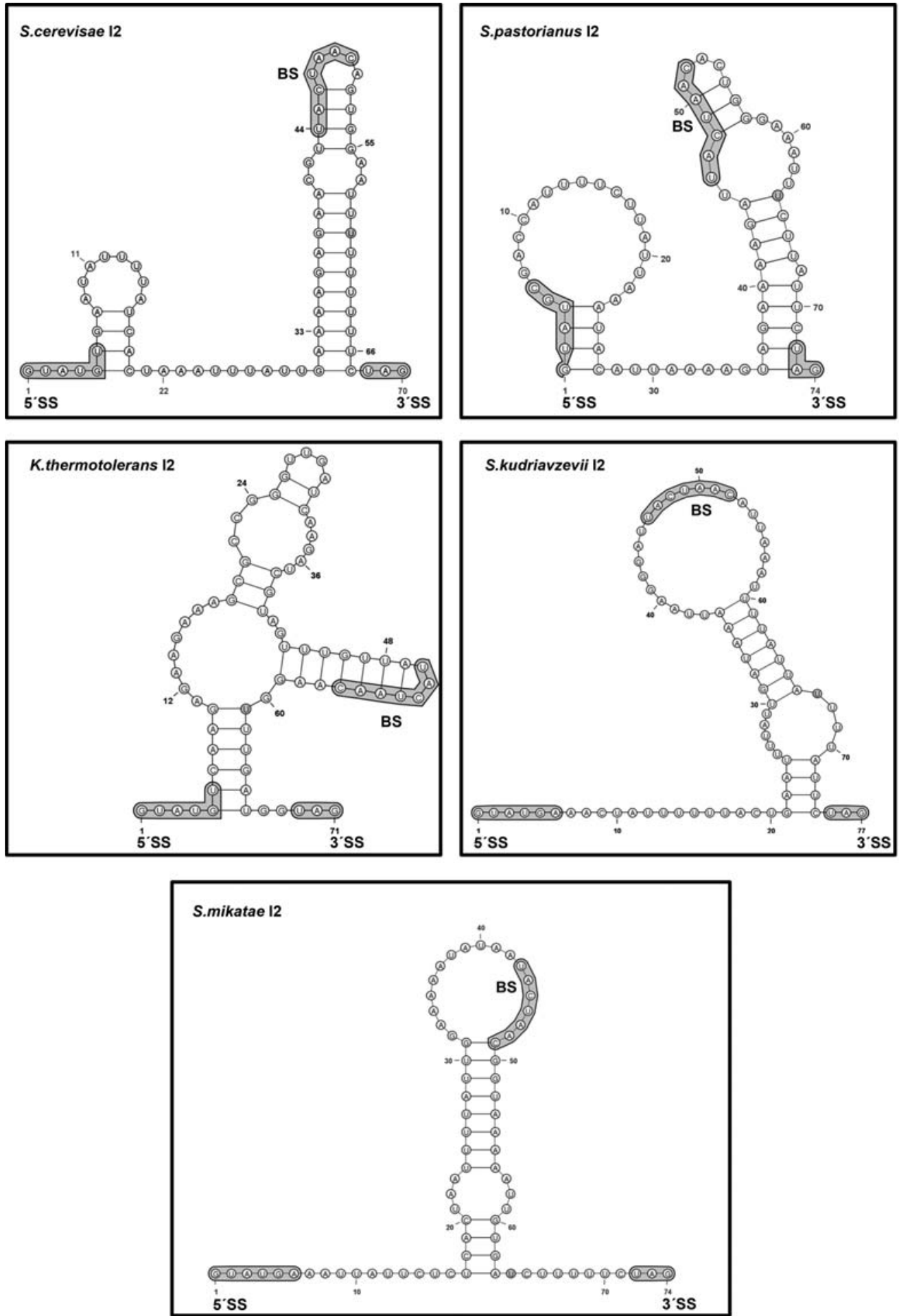

FIGURE 1. Predicted secondary structure of SUS1 intron 2 RNA. Minimum free-energy I2 structures in S. cerevisiae, S. pastorianus, K. thermotolerans, S. kudriavzevii, and S. mikatae. The $5^{\prime}$ SS, 3'SS, BS sequences and the uridine at -9 position preceding the AG are highlighted. The predictions were carried out with Mfold.

pSUS1-I2-mut1-CUP1 (with a wild-type I1 and a shortened I2 hairpin). Splicing was monitored both by assessing copper tolerance and by semiquantitative reverse transcription PCR (semi-qRT-PCR). Partial deletion of the stem of the I2 hair- pin led to a clear reduction in copper tolerance compared with wild-type I2 (Fig. 3B), suggesting that the I2 hairpin was important for SUS1 expression. When the different SUS1 splice forms generated from expressing I2-mut1 in sus $1 \Delta$ 
A

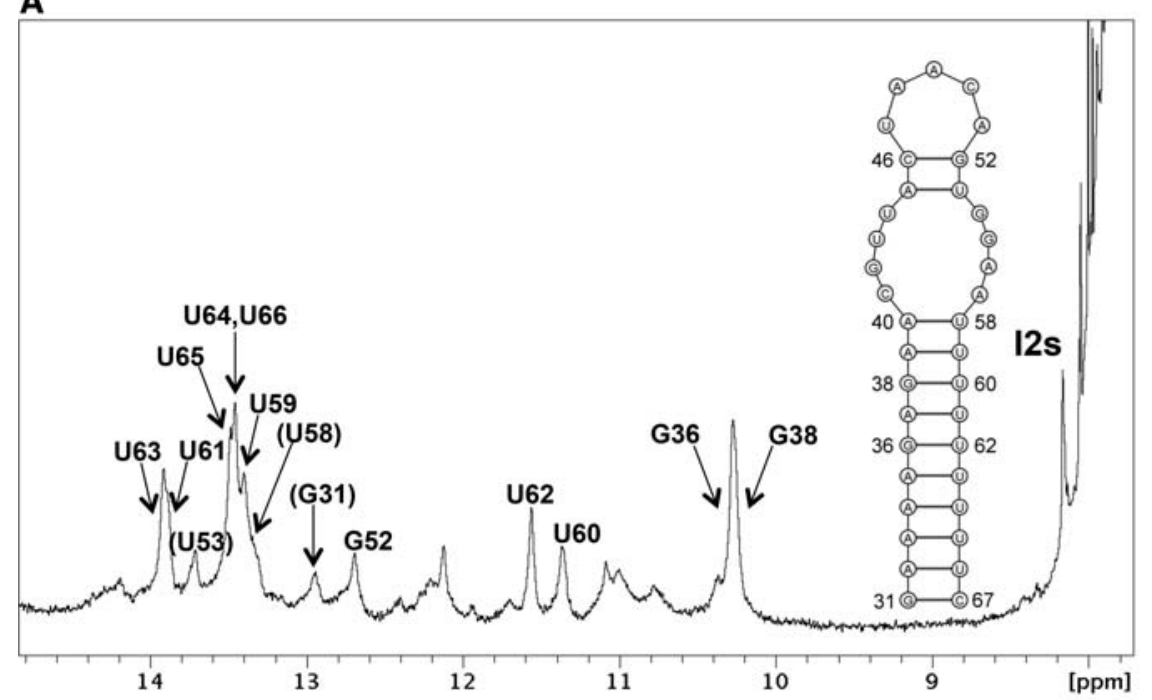

B

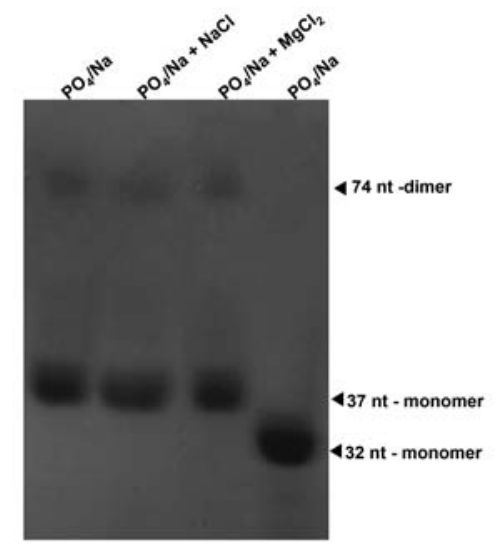

C

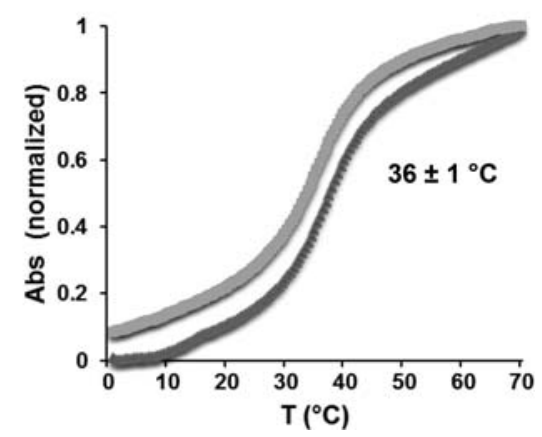

FIGURE 2. In vitro analysis of intron 2 structure and stability. (A) Imino proton NMR spectrum and NMR-supported secondary structure of the 37-nt I2s oligomer. Conditions: $7^{\circ} \mathrm{C}, 2 \mathrm{mM}$ sodium phosphate ( $\mathrm{pH} \mathrm{6.0)}$ ) and $100 \mathrm{mM} \mathrm{NaCl}$. The assignments (parentheses indicate tentative assignments) were obtained from NOESY and TOCSY spectra in $\mathrm{H}_{2} \mathrm{O}$ and $\mathrm{D}_{2} \mathrm{O}$. (B) Native gel comparing the electrophoretic mobility of $16 \mu \mathrm{M}$ I2s samples previously annealed in different ionic conditions: (i) $2 \mathrm{mM}$ sodium phosphate ( $\mathrm{pH} 6.0$ ), (ii) $2 \mathrm{mM}$ sodium phosphate and 100 $\mathrm{mM} \mathrm{NaCl}$, (iii) $2 \mathrm{mM}$ sodium phosphate, and $2 \mathrm{mM} \mathrm{MgCl}_{2}$. Lane 4 contained a 32 -nt RNA hairpin control. $(C)$ UV-monitored thermal denaturation curve of I2s in $2 \mathrm{mM}$ sodium phosphate ( $\mathrm{pH}$ 6.0) and $100 \mathrm{mM} \mathrm{NaCl}$. The average melting temperature of I2s under these ionic conditions is indicated in the graph.

cells were measured by semi-qRT-PCR, the accumulation of different pre-mRNAs containing I2, I1 or both introns was evident (Fig. 3C). In agreement with the results of the copper assay, semi-qRT-PCR experiments indicated a reduced amount of fully spliced mRNA relative to the wild-type cultures (Fig. 3D). Since I1-containing isoforms also accumulated with the I2-mut1 mutant (Fig. 3C), we generated an I2-mut1 construct lacking I1 (I2-mut1 $\Delta \mathrm{I} 1$ ). The absence of I1 did not change the effect of I2-mut1 on SUS1 processing as evidenced by cell growth in coppercontaining plates (Supplemental Fig. S3). This demonstrated that the splicing effects caused by the I 2 mutation did not depend on I1.

A fast-migrating SUS1 transcript was detected in the semi-qRT-PCR gels of I2-mut1 (Fig. 3C, last lane). Sequencing of this band revealed that it corresponded to the SUS1 E1-E3 transcript, which was already observed (Hossain et al. 2011). Partial deletion of the double-helical stem of the I2 hairpin promoted accumulation of I1- and I2-containing pre-mRNAs (Fig. 3C). The poor splicing efficiency of I2-mut1 could have led to increased exon 2 skipping, which contributes to alternative splicing and the production of the E1-E3 isoform (discussed below).

\section{Stabilization of the intron 2 hairpin affects SUS1 expression}

The functional relevance of the I2 hairpin was further studied by analyzing three new structural mutants. Mutant I2mut2 was designed to disrupt the 10-bp stem at the base of the I 2 hairpin by replacing the 10 -nt $\mathrm{G}_{31} \mathrm{AAAAGAGAA}_{40}$ purine stretch with $\mathrm{C}_{31}$ UUUUUUUUU ${ }_{40}$ (Fig. $4 \mathrm{~A}$, left panel). In contrast, I2-mut3 was intended to stabilize this 10-bp stem by converting its seven A:U pairs into G:C pairs: thus the As of the $\mathrm{G}_{31} \mathrm{AAAAGAGAA}_{40}$ purine stretch were replaced with Gs, and the opposing Us in the $\mathrm{U}_{58} \mathrm{UUUUU} \mathrm{UUUUC}_{67}$ pyrimidine segment $\left(\mathrm{U}_{58}-\mathrm{U}_{59}, \mathrm{U}_{61}\right.$, and $\left.\mathrm{U}_{63}-\mathrm{U}_{66}\right)$ with Cs (Fig. 4A, middle panel). On the other hand, I2-mut4 was aimed to disrupt the base pairs affecting BS nucleotides without altering the lower doublehelical stem of the hairpin. To achieve this, the $\mathrm{G}_{52} \mathrm{UGG}_{55}$ segment was replaced with $\mathrm{C}_{52} \mathrm{CUU}_{55}$ (Fig. 4A, right panel). Note that none of these I2-mut2, I2mut3, or I2-mut 4 mutants affected the size of the intron, the number of nucleotides separating the BS and the $3^{\prime} \mathrm{SS}$, or the PPT following the BS. The effect of these mutants on the structure and stability of the I2 hairpin was assessed with Mfold calculations (Fig. 4A; Supplemental Fig. S4A) as well as with gel electrophoresis and thermal denaturation analyses of mutant 37-nt I2s oligonucleotides (Supplemental Fig. S4B,C). In the predicted structure of I2-mut3, G:C pairs replaced the seven A: $U$ pairs of the stem at the base of the I2 hairpin. In agreement with this, the I2-mut3s oligomer formed a highly stable monomeric structure with a $T_{\mathrm{m}}$ value of $86^{\circ} \mathrm{C}$ (Supplemental Fig. S4B), more than two times higher than the $T_{\mathrm{m}}$ of wild-type $\mathrm{I} 2 \mathrm{~s}$, which melted at $36^{\circ} \mathrm{C}$ in the 
A

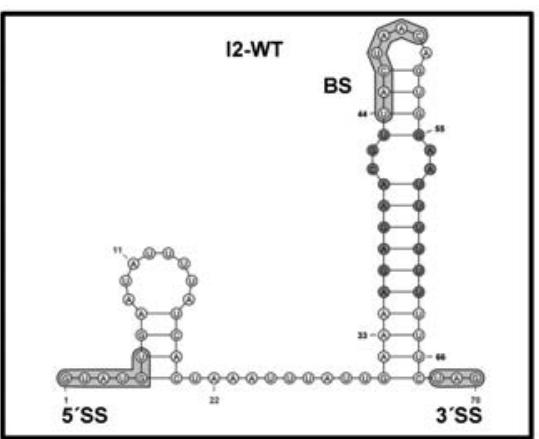

B

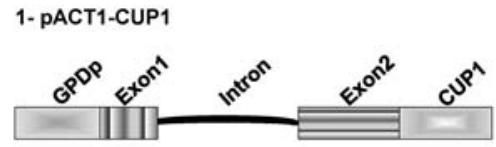

2-pSUS1-WT-CUP1
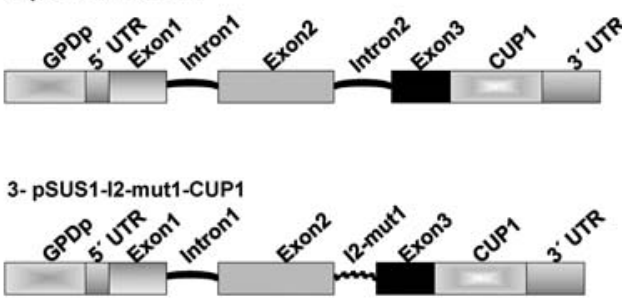

C

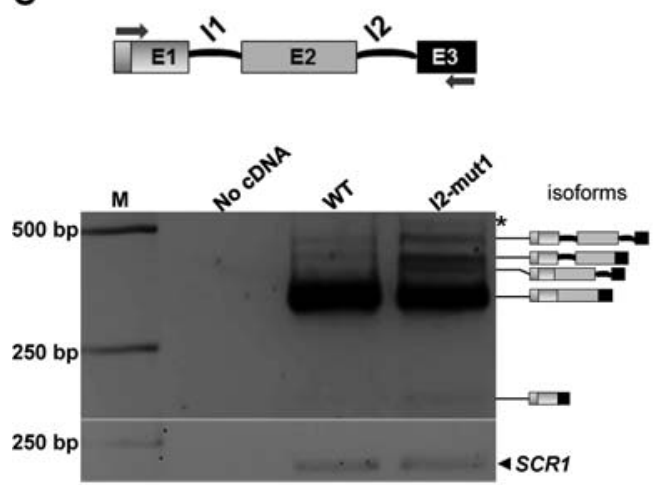

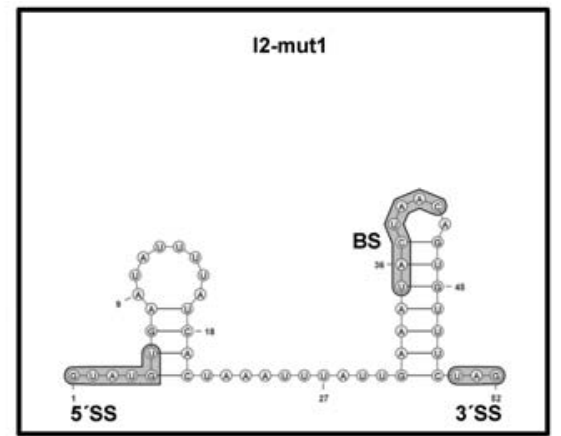

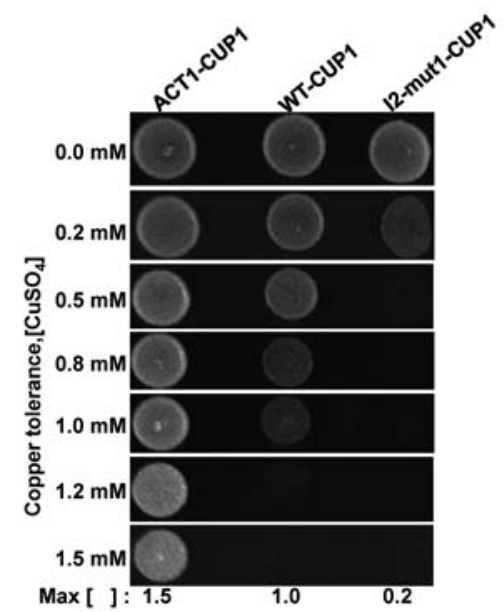

D
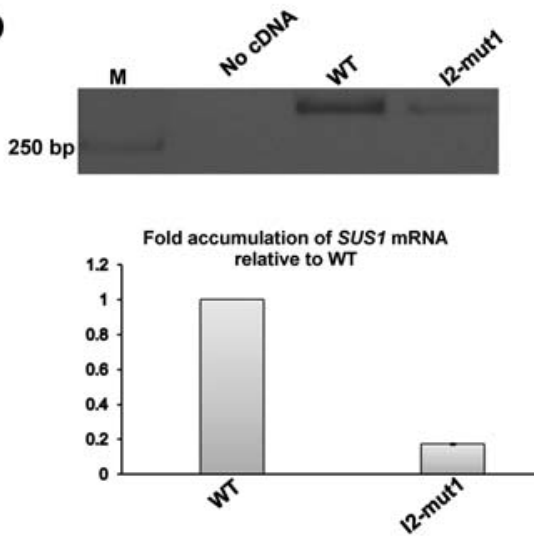

FIGURE 3. Partial deletion of the I2 hairpin double-helical stem affects SUS1 splicing. (A) MFold-predicted secondary structure of wild-type I2. The nucleotides highlighted in dark gray were removed to construct the intron 2 deletion mutant 1 (I2-mut1). The prediction of I2-mut 1 secondary structure is shown on the right. (B) Copper assay of cup1 $\Delta$ cells transformed with plasmids containing SUS1g-CUP1, SUS1-I2-mut1-CUP1, and ACT1CUP1 as a control. Maximum copper tolerance is indicated. (C) Semi-q-RT-PCR (35 cycles) to amplify SUS1 transcripts resolved in a 3\% agarose gel from cells expressing SUS1g-CUP1 (WT) or SUS1-I2-mut1-CUP1 (I2-mut1) in each case. A PCR without cDNA (no cDNA) was included as control. The stick diagrams on the right of the gel show the different isoforms of SUS1 RNA. (D) Semi-q-RT-PCR (25 cycles) to amplify SUS1 transcripts resolved in a 3\% agarose gel from cells expressing SUS1g-CUP1 (WT) or SUS1-I2-mut1-CUP1 (I2-mut1) in each case (upper panel). qPCR showing mRNA accumulation from cells expressing SUS1g-CUP1 (WT) or SUS1-I2-mut1-CUP1 (I2-mut1) normalized to the amount of SCR1ncRNA (lower panel). Error bars represent SE for at least three independent experiments ([*] Spurious PCR product).

same ionic conditions (Fig. 2). In the predicted structure of the full-length I2-mut2 intron the 10-bp lower stem was completely eliminated and the BS lied in a fully unstructured region (Fig. 4A). Although the PPT nucleotide formed a small hairpin whose terminal pairs partially encompassed the $3^{\prime} \mathrm{SS}$, these terminal U:G and A:U pairs showed significant positional entropy in the ensemble of theoretical structures (data not shown) and are likely unstable. In agreement with these predictions, the I2-mut2s oligonucleotide gave rise to delayed electrophoretic bands and did not exhibit 
A
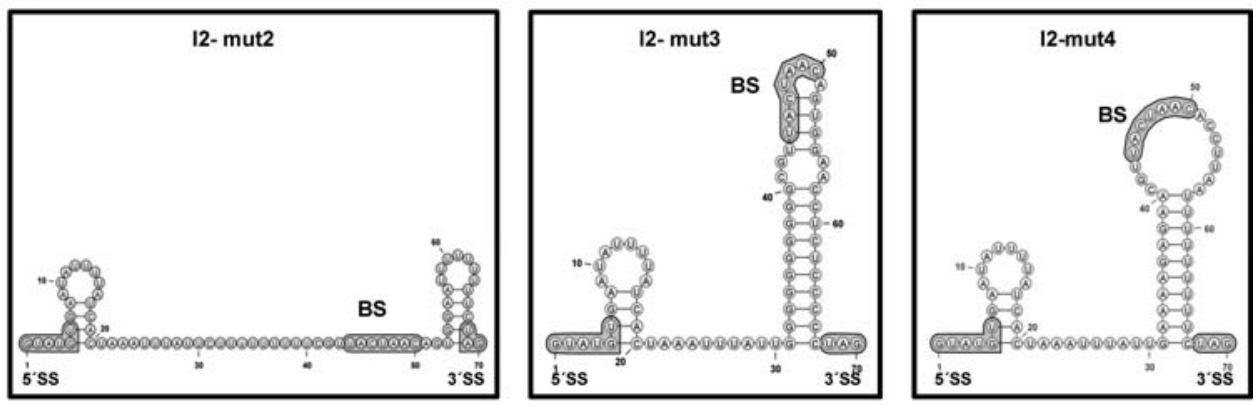

B
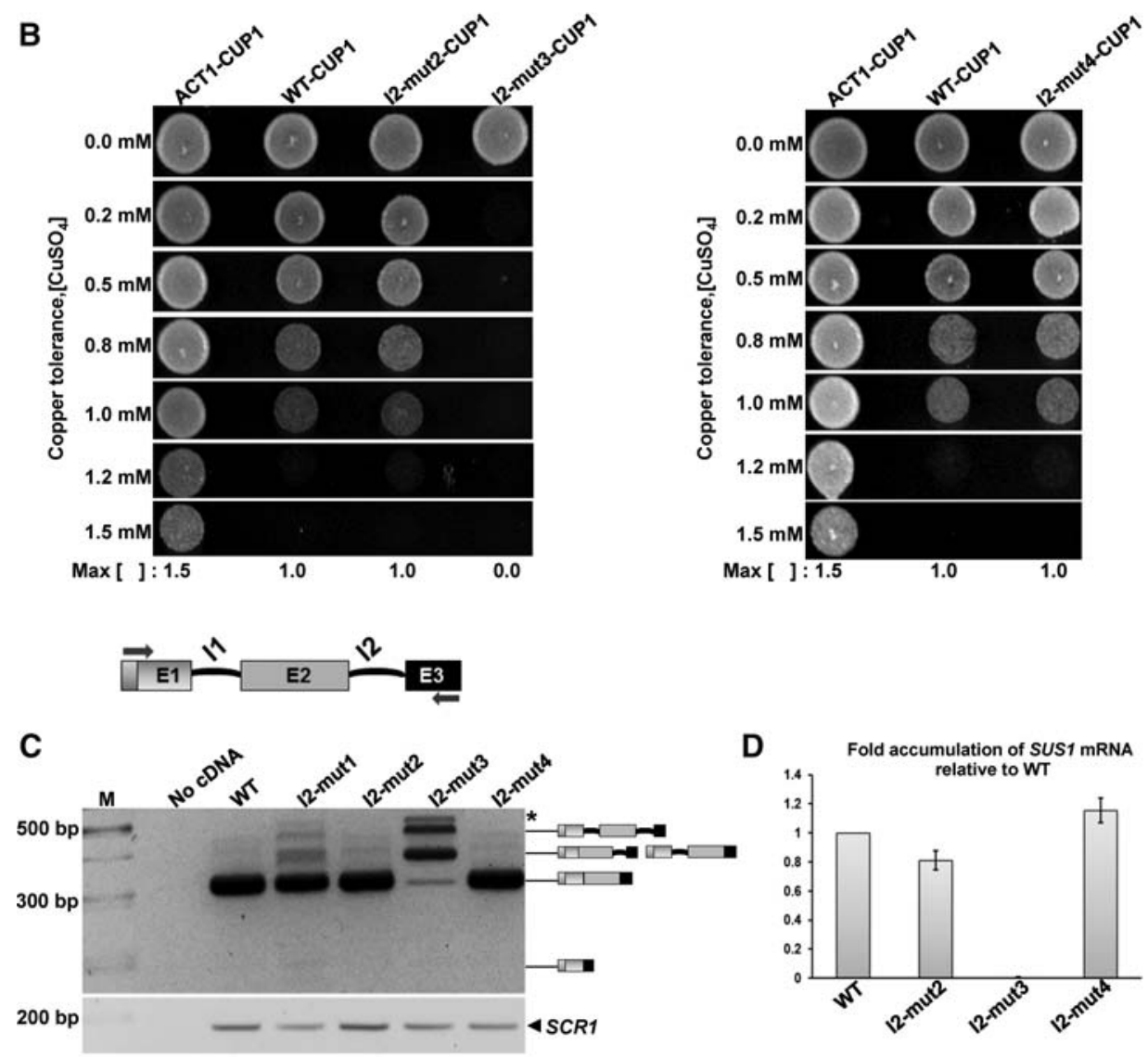

FIGURE 4. Stabilization of the intron 2 hairpin affects SUS1 expression. (A) MFold-predicted secondary structure of the I2 hairpin disruption mutant (I2-mut2, left panel), hairpin stabilization mutant (I2-mut3, middle panel) and BS base-pairing disruption mutant (I2-mut4, right panel). The nucleotides highlighted in gray were substituted to generate each mutant. (B) Copper assay of cup1 $1 \Delta$ cells transformed with plasmids containing SUS1g-CUP1, SUS1-I2-mut2-CUP1, SUS1-I2-mut3-CUP1, SUS1-I2-mut4-CUP1, and ACT1-CUP1 as a control. Maximum copper tolerance is indicated. (C) Semi-q-RT-PCR (35 cycles) to amplify SUS1 transcripts resolved in a 3\% agarose gel from cells expressing SUS1g-CUP1 (WT) or SUS1I2-mut1-CUP1 (I2-mut1), SUS1-I2-mut2-CUP1 (I2-mut2), SUS1-I2-mut3-CUP1 (I2-mut3), and SUS1-I2-mut4-CUP1 (I2-mut4) in each case. A PCR without cDNA (no cDNA) was included as a control. The stick diagrams on the right of the gel show the different isoforms of SUS1 RNA. (D) qPCR showing mRNA accumulation from cells expressing SUS1 as in (C) normalized to the amount of SCR1 ncRNA. Error bars represent SE for at least three independent experiments ([*] Spurious PCR product).

any detectable transition in UV-monitored thermal denaturation experiments (Supplemental Fig. S4), indicating the formation of a very unstable structure or no structure at all. In the I2-mut4 predicted fold, the BS nucleotides did not form any base pairs in the apical region of the hairpin while the AU-rich lower stem remained unaffected (Fig. 4A;
Supplemental Fig. S4A). In accordance with this, I2-mut4s formed a monomeric structure with a slightly reduced $T_{\mathrm{m}}$ value relative to wild-type $\mathrm{I} 2 \mathrm{~s}\left(32^{\circ} \mathrm{C}\right.$ versus $36^{\circ} \mathrm{C}$ in the presence of $100 \mathrm{mM} \mathrm{NaCl}$ ) (Supplemental Fig. S4).

We then evaluated the impact of each of these structural mutants on SUS1 expression as described above for 
I2-mut1. The I2-mut2, I2-mut3, and I2-mut4 mutants were first cloned in the CUP1 reporter system and transformed into cup $1 \Delta$ cells. As shown in Figure 4B, left panel, I2-mut3 drastically impaired cell growth relative to wild type, indicating that stabilization of the I2 stem-loop decreased SUS1 expression. In contrast, I2-mut 2 and I2-mut 4 did not impair cell growth when compared to wild type (Fig. 4B, left and right panels, respectively). To assess the production of SUS1 isoforms by these mutants, semi-q-RT-PCR was carried out to amplify SUS1 transcripts in sus1D cells bearing mutant constructs. In agreement with the copper assay observations, the I2-mut3 mutant stabilizing the stem-loop structure completely inhibited I 2 splicing, leading to a clear enrichment of I2- and I1-I2-containing pre-mRNA SUS1 transcripts (Fig. 4C). The I2-mut2 and I2-mut4 mutant sequences did not lead to a detectable increment of $\mathrm{I} 1$ or $\mathrm{I} 2$ retention in the gels (Fig. 4C), but semi-q-RT-PCR analyses of I2-mut2 transcripts showed a slight reduction of fully spliced SUS1
mRNA relative to wild type (Fig. 4D), while a small increase was observed in the case of I2-mut4 (Fig. 4D).

\section{High-temperature effects on SUS1 splicing upon intron 2 structural mutations}

Interesting differences were observed between the structural mutants when the experiments were carried out under stress. Previous analyses with wild-type cells incubated at $42^{\circ} \mathrm{C}$ revealed reduction of SUS1 expression, accompanied by accumulation of unspliced SUS1 transcripts and decrease of fully spliced mRNA (Cuenca-Bono et al. 2011). We tested the effect of the different structural mutants in these conditions by semi-q-RT-PCR (Fig. 5A). The most drastic variation relative to standard conditions (cells growing at $30^{\circ} \mathrm{C}$ ) was observed for mutants I2-mut1 and I2-mut3, which, compared with the wild-type sequence, generated a large accumulation of transcripts containing both introns, together with a drastic
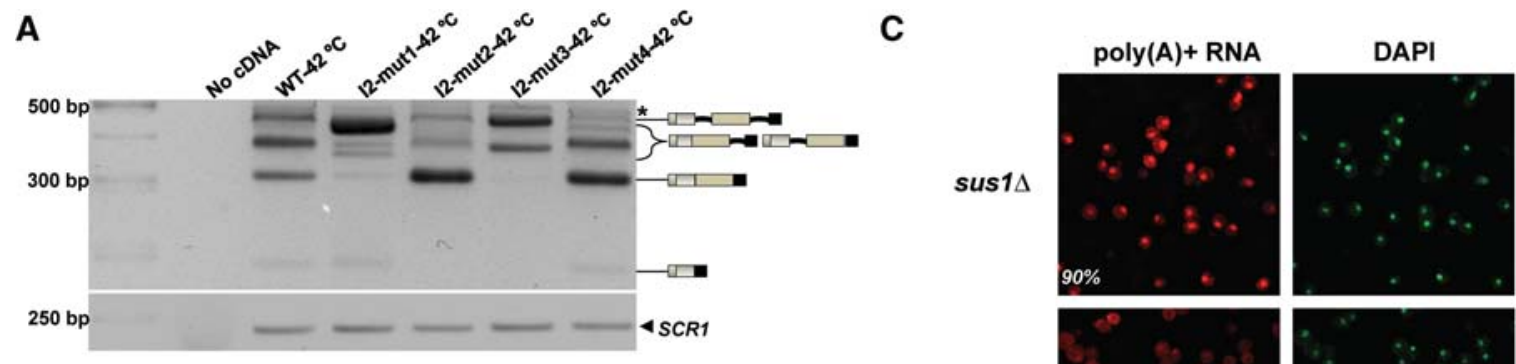

B
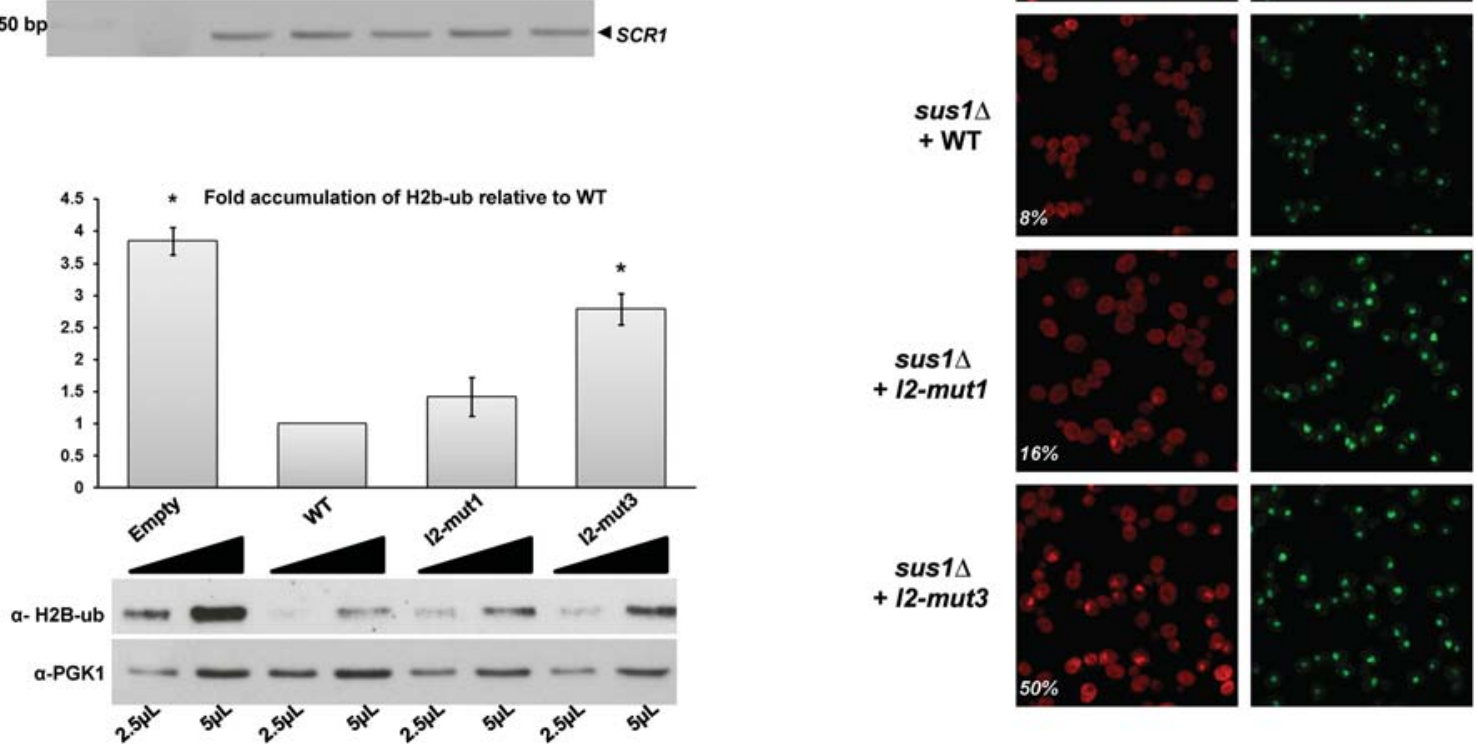

sus1A

+ I2-mut3
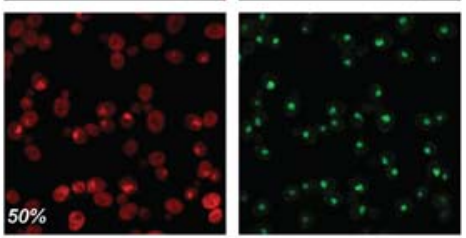

FIGURE 5. Deletion or stabilization of intron 2 hairpin affects Sus1 cellular roles. (A) Analysis of SUS1 transcripts by semi-q-RT-PCR from cells expressing SUS1g (WT) or I2 structural mutants (SUS1-I2-mut1-CUP1 (I2-mut1), SUS1-I2-mut2-CUP1 (I2-mut2), SUS1-I2-mut3-CUP1 (I2mut3), and SUS1-I2-mut4-CUP1 (I2-mut4) from cells incubated at $42^{\circ} \mathrm{C}$ for $20 \mathrm{~min}$. Bands corresponding to the different transcripts are indicated. A PCR without cDNA (no cDNA) was included as control. (B) Quantification and representative blots obtained with increasing concentration of whole-cell extracts (WCE) from sus1 $\Delta$ cells transformed with an empty plasmid (Empty), or with plasmids expressing SUS1g (WT), SUS1-I2mut1 (I2-mut1), or SUS1-I2-mut3 (I2-mut3). Two volumes $(2.5 \mu \mathrm{L}$ or $5 \mu \mathrm{L})$ of each WCE were resolved by $15 \%$ SDS-polyacrylamide gel, transferred to a nitrocellulose membrane. The membrane was cut and the upper part was incubated with PGK1 antibody (loading control) and the lower with H2B-ub antibody. Bar graph shows total H2B-ub levels normalized to PGK1 after quantification of Western blot signals. Similar results were obtained using total H2B as a loading control (data not shown). Error bars represent $\mathrm{SE}$ for at least three independent experiments. $(C)$ Representative images of poly $(\mathrm{A})^{+}$RNA localization in sus $1 \Delta$ cells, transformed as in $(B)$ and assayed by in situ hybridization using Cy3-labeled oligo(dT) probes. The percentages indicate the number of cells showing mRNA accumulation and correspond to the averages of at least three independent biological replicates. DAPI staining to mark the nucleus is shown on the right. Cells were incubated at $37^{\circ} \mathrm{C}$ in rich media for $2 \mathrm{~h}$. 
reduction of fully spliced mRNA. At $42^{\circ} \mathrm{C}$, I2-mut2 and I2-mut4 exhibited less intron retention as well as increased levels of fully spliced mRNA relative to wild type. In addition, I2-mut2 clearly showed less I1 retention relative to wild type or I2-mut4 (Fig. 5A). This confirmed that I2 splicing can affect an upstream splicing step, supporting the results obtained with mutant I2-mut1.

\section{Sus1 functions in histone deubiquitination and mRNA export are affected in 12 hairpin mutants that inhibit splicing}

Sus1 is a conserved factor that is required for mRNA export and histone $\mathrm{H} 2 \mathrm{~B}$ deubiquitination, and deletion or mutation of SUS1 has been shown to impair mRNA export and histone H2B deubiquitination (Rodríguez-Navarro et al. 2004; Köhler et al. 2006; Kurshakova et al. 2007; Zhao et al. 2008; Klöckner et al. 2009; Kopytova et al. 2010; Galan and Rodríguez-Navarro 2012; García-Oliver et al. 2012). So far, our analyses have shown that partial deletion (I2-mut1) and stabilization (I2-mut3) of the 37-nt hairpin structure formed by intron 2 led to differential SUS1 isoform expression. These changes in splicing could lead to a deregulation of Sus1 protein production. To study whether these I2 mutations had functional consequences, cells lacking the SUS1 gene (sus1 $\Delta$ ) were transformed with wild-type and I2-mutant SUS1 plasmids, and their ability to complement SUS1 deletion phenotypes was evaluated. As shown in Figure 5B, I2-mut3 was unable to deubiquitinate histone $\mathrm{H} 2 \mathrm{~B}$ at wildtype levels, in agreement with the low expression of fully spliced SUS1 isoform in these cells (Fig. 4C). Although I2-mut1 likewise reproducibly reduced the amount of fully spliced SUS1 (Fig. 3C,D), the reduction of ubiquitinated $\mathrm{H} 2 \mathrm{~B}$ relative to WT was not statistically significant (Fig. 5B). Similar results were obtained using total $\mathrm{H} 2 \mathrm{~B}$ as a loading control (data not shown). Concomitantly, defects in mRNA export provoked by SUS1 deletion observed in $90 \%$ of the cells (Fig. 5C, left panel) were more apparent for I2-mut3 than I2-mut 1: 50 and 16\% of the cells transformed with these two mutants, respectively, accumulated mRNA, relative to $8 \%$ transformed with wild-type SUS1 (Fig. 5C).

\section{DISCUSSION}

The structure of RNA molecules plays an important role during their metabolism, adding a new layer of regulation to different steps of the gene expression pathway. In this study, we have analyzed the impact on splicing of a hairpin structure formed by the second intron of the pre-mRNA of $S$. cerevisiae SUS1. Secondary structure predictions of this intron in this and other yeast species revealed the formation of a stemloop structure with BS nucleotides located near the apical loop and $3^{\prime} \mathrm{SS}$ nucleotides close to the $3^{\prime}$ terminus of the base-paired stem (Fig. 1). In several cases, $5^{\prime}$ SS nucleotides were likewise predicted to form base pairs with downstream nucleotides that might affect splicing efficiency. However, our study focused on the hairpin formed by the BS and 3'SS segment, leaving the 5'SS region unmodified. In all species in which the I2 hairpin was present a pyrimidine-rich sequence was located between the BS and the $3^{\prime}$ SS. This polypyrimidine tract (PPT) has been shown to be important for splicing in higher eukaryotes (Roscigno et al. 1993; Liu and Mertz 1996; Coolidge et al. 1997). In contrast, in yeast species the 5'SS, BS, and 3'SS sequences are highly conserved, but the PPTs are not (Rymond and Rosbash 1985; Fouser and Friesen 1987). Interestingly, the PPT of SUS1 I2 seemed to be partially conserved in yeast, and a uridine at position -9 preceding the 3'SS was present in all cases (Fig. 1). It has been suggested that splicing in fungi may be different from that in vertebrates and may require additional proteins that interact with PPTs upstream of the BS (Kupfer et al. 2004). However, other studies have indicated that PPTs downstream from the BS do provide a positioning function (Matlin et al. 2007). Furthermore, nonconserved sequences can also influence alternative splice site selection via structural motifs, supporting the idea that pre-mRNA introns can be highly evolved molecules with functional and structural constraints (Deshler and Rossi 1991).

To confirm the theoretical predictions, we studied the structure and stability of $S$. cerevisiae I2, represented by a 37-nt I2s oligonucleotide encompassing the predicted hairpin. The NMR data indicated that I2s formed a hairpin structure in solution containing a 32-nt double-helical stem and a 5-nt UAACA apical loop containing the last $4 \mathrm{nt}$ of the BS (Fig. 2). The stem comprised an AU-rich tract of 10 base pairs interrupted by a CGUU:GGAA internal loop that was separated from the apical loop by two Watson-Crick pairs. The UV-thermal denaturation experiments showed that this intron 2 hairpin had surprisingly low thermal stability. It is well established that both the BS and the PPT of the introns need to be single-stranded to allow binding of the BBP (SF1 in mammals) and Mud2 $\left(\mathrm{U}_{2} \mathrm{AF}_{65}\right)$ proteins in the first steps of spliceosome assembly (Gahura et al. 2011; Pérez-Valle and Vilardell 2012). It is therefore remarkable that two of the BS nucleotides $\left(\mathrm{A}_{45}\right.$ and $\left.\mathrm{C}_{46}\right)$ and the complete PPT of I2 are contained in the base-paired stem of the I2 hairpin. This observation, together with the UV thermal denaturation and NMR spectroscopy findings showing that the structure was thermally labile, supported a regulatory role for the I2 hairpin on splicing.

To assess how important I2 hairpin formation was for SUS1 function and regulation, four structural mutants were analyzed: I2-mut 1 (containing an I2 hairpin with a shortened double-helical stem), I2-mut2 (with a destabilized stem), I2mut3 (stabilized stem), and I2-mut4 (with an intact stem and an opened apical BS loop) (Figs. 3, 4). The phenotypes observed when partially deleting the stem of the I2 hairpin (I2-mut1) suggested that this stem-loop structure was important for SUS1 expression. However, since part of the PPT was deleted we cannot exclude the possibility that this 
could have affected SUS1 splicing. Notably, I1 retention increased with the I2-mut1 mutation (Fig. 3C). Previous studies proposed that the upstream intron of SUS1 modulated the processing of I2 (Hossain et al. 2011). Our results demonstrate that intrinsic features of I2 can also lead to I1 retention, suggesting that the downstream intron affected splicing of its upstream counterpart.

The most drastic effects were observed with the I2-mut3 mutant. The corresponding I2-mut3s oligomer formed a highly stable structure with a melting temperature more than two times higher than that of the wild-type I2s hairpin. This mutation completely inhibited I2 splicing, likely due to the inability of the spliceosome components to act in this environment. The mutants that completely destabilized the double-helical stem of the I2 hairpin (I2-mut2) or further opened the apical BS loop (I2-mut4) did not provoke a detectable increment of I1 or I2 retention, but behaved differently in terms of the amount of fully spliced SUS1 mRNA generated. The I2-mut4 transcripts showed a slight increase of fully spliced SUS1 mRNA relative to wild type, while a small reduction was observed in the case of I2-mut2. The small positive effect of I2-mut 4 may be attributed to the marginally greater accessibility of BS nucleotides in this sequence. This would be consistent with the NMR analyses of the wildtype hairpin, which indicated the presence of only two stable base pairs in this region (Fig. 2), and with the slight reduction in melting temperature $\left(4^{\circ} \mathrm{C}\right)$ observed for $\mathrm{I} 2$-mut 4 s relative to the wild-type oligomer (Fig. 2; Supplemental Fig. S4C). Like I2-mut1, I2-mut2 was detrimental for splicing probably because the presence of the lower double-helical stem of the I2 hairpin increases the accessibility of BS and 3'SS nucleotides to splicing factors. However, the slight reduction of fully spliced mRNA observed for I2-mut2, where the double-helical stem of the hairpin was disrupted, was much smaller than that observed for I2-mut1, containing a shortened stem. It is worth noting in this regard that in the I2-mut2 sequence an artificial PPT upstream of the BS was generated (Fig. 4A), which could promote splicing (Kupfer et al. 2004). Thus, positioning the BS in between two strong PPTs may have compensated the splicing defects caused by the destabilization of the I2 hairpin, explaining the smaller effect observed for I2-mut2 relative to I2-mut1.

The functional relevance of the I2 hairpin was further studied by analyzing the ability of the I2-mut1 and I2-mut3 mutants to accomplish Sus1 protein functions. The effect of these mutations on deubiquitination and mRNA export (Fig. 5) suggests that the reduced amount of Sus1 protein likely produced by these mutants is still enough to partially complement Sus1 functions, including sus $1 \Delta$ temperature sensitive growth defect (data not shown). This is interesting, since overexpression of Sus1 has been shown to largely impair its functionality (Cuenca-Bono et al. 2011; Hossain et al. 2011).

In conclusion, we have demonstrated the existence of a weakly stable, 37-nt hairpin structure formed by the second intron of S. cerevisiae SUS1 that contains BS nucleotides in its apical loop and $3^{\prime}$ SS nucleotides immediately after the $3^{\prime}$ terminus of the stem. Our study shows that this structure regulates the expression of SUS1 isoforms by influencing SUS1 splicing. The effect of different mutants modulating the structure and stability of the hairpin suggests that this I2 stem-loop likely increases the accessibility of BS and 3'SS nucleotides to the splicing components by maintaining the BS in a readily accessible and easily opened apical loop environment and the $3^{\prime}$ SS nucleotides unstructured immediately after the double-helical stem. In this way, the efficiency of I 2 removal would be regulated by the formation of the structure.

Moreover, the effect of mutants I2-mut1 and I2-mut2 on I1 retention indicates that the $\mathrm{I} 2$ hairpin also modulates the splicing of the upstream intron. In this regard, it may be possible that the weakly stable hairpin has evolved as a mechanism to coordinate removal of I1, whose splicing is very inefficient and needs more time to occur. On the other hand, exon 2 skipping was promoted by mutant $\mathrm{I} 2$-mut 1 under standard conditions. SUS1 exon 2 skipping was previously detected in wild-type cells when different components of the splicing machinery (MSL1, LEA1, and MUD2) were deleted, and was explained by the role of these factors in recognizing the suboptimal BS of I1 (Hossain et al. 2009; Will and Lührmann 2011). Interestingly, our results indicate that the presence of these factors is not sufficient to supress exon skipping when intron 2 is mutated. This opens the possibility that I2-dependent mechanisms also contribute to regulate exon skipping.

\section{MATERIALS AND METHODS}

\section{Sequences and structure prediction of SUS1}

The sequences of the second intron of SUS1 across different species of yeast were obtained from the Yeast Genome Database (SGD) and NCBI (http://www.ncbi.nlm.nih.gov/). The secondary structure predictions were carried out with the MFOLD web server (http ://mfold.rna.albany.edu/?q=mfold/rna-folding-form) (Zuker 2003), and the structures were drawn using VARNA (http://varna.lri.fr/) (Darty et al. 2009).

\section{Large-scale RNA transcription}

The wild-type I2s and mutant I2-mut2s, I2-mut3s, and I2-mut4s RNA oligomers used for NMR spectroscopy, gel electrophoresis, and UV thermal experiments were prepared by T7-polymerase in vitro transcription using synthetic oligonucleotide DNA templates. The RNA transcripts were purified on denaturing gels containing $20 \%$ acrylamide and $8 \mathrm{M}$ urea. After electroelution from the gel, the RNAs were ethanol-precipitated two times and desalted with Sephadex G-25 cartridges. The I2s NMR samples were microdialyzed in aqueous solutions containing $2 \mathrm{mM}$ sodium phosphate ( $\mathrm{pH}$ 6.0) and $0.1 \mathrm{mM}$ EDTA with no added salts or additionally containing $100 \mathrm{mM} \mathrm{NaCl}$. The RNA concentration in these samples ranged between 0.10 and $0.15 \mathrm{mM}$. 


\section{NMR spectroscopy}

NMR spectra were acquired on $600 \mathrm{MHz}$ (cryoprobe-equipped) and $500 \mathrm{MHz}$ Bruker Avance III spectrometers, and analyzed using Topspin 1.3 (Bruker Biospin) and Sparky 3.110 (T.D. Goddard, D.G. Kneller, UCSF USA, 2004). The I2s systems were studied using two-dimensional WATERGATE-NOESY (with 150-400 ms mixing times) and WATERGATE-TOCSY experiments (60-msec mixing time) recorded in $90 \% \mathrm{H}_{2} \mathrm{O} / 10 \% \mathrm{D}_{2} \mathrm{O}$ at several temperatures (between $7^{\circ} \mathrm{C}$ and $\left.17^{\circ} \mathrm{C}\right)$. TOCSY and NOESY $(250 \mathrm{msec})$ experiments were also acquired in $\mathrm{D}_{2} \mathrm{O}$ at $13^{\circ} \mathrm{C}$ and $23^{\circ} \mathrm{C}$. The recycle delay was $2 \mathrm{sec}$ in all cases.

\section{UV thermal denaturation}

The thermal stability of wild-type I2s and mutant I2-mut2s, I2mut3s, and I2-mut4s oligomers was monitored by measuring the UV absorbance at $260 \mathrm{~nm}$ as a function of temperature in a Varian Cary $100 \mathrm{UV} / \mathrm{VIS}$ spectrophotometer. The temperature was raised from $0^{\circ} \mathrm{C}-10^{\circ} \mathrm{C}$ to $70^{\circ} \mathrm{C}-100^{\circ} \mathrm{C}$ at a gradient of $0.5^{\circ} \mathrm{C}-$ $2.0^{\circ} \mathrm{C} \mathrm{min}^{-1}$ and subsequently decreased at the same rate to evaluate the reversibility of the process. The experiments were carried out using $0.4-0.5 \mathrm{ODU} / \mathrm{mL}$ of RNA $(1.0-1.3 \mu \mathrm{M})$. The thermal denaturing profiles of the wild-type I2s sequence were examined in the following ionic conditions: $2 \mathrm{mM}$ sodium phosphate ( $\mathrm{pH}$ 6.0) with no additional salts, or containing either $100 \mathrm{mM} \mathrm{NaCl}$ or $2 \mathrm{mM} \mathrm{MgCl}_{2}$. The mutant oligomers were studied in $2 \mathrm{mM}$ sodium phosphate (pH 6.0) and $100 \mathrm{mM} \mathrm{NaCl}$. All melting experiments were repeated at least two times in each ionic condition. Before each experiment, RNA samples were heated at $95^{\circ} \mathrm{C}$ for $\sim 5 \mathrm{~min}$ and immediately placed on ice for $5 \mathrm{~min}$.

\section{Gel electrophoresis}

These experiments were used to assess the strand stoichiometry of the structures formed by the I 2 oligonucleotides, as well as to evaluate the number of structural species formed by each sequence in a given condition. Native gels were run at $4^{\circ} \mathrm{C}$ for $\sim 14 \mathrm{~h}$ under constant voltage $(90 \mathrm{~V})$. We used 20\% 19:1 acrylamide:bisacrylamide gels and $89 \mathrm{mM}$ Tris-Borate (TB) as running buffer. These experiments involved 13-18 $\mu \mathrm{M}$ wild-type or mutant I2s samples, previously annealed as specified above in the following ionic conditions: $2 \mathrm{mM}$ sodium phosphate ( $\mathrm{pH}$ 6.0) with no additional salts, or containing either $100 \mathrm{mM} \mathrm{NaCl}$ or $2 \mathrm{mM} \mathrm{MgCl}_{2}$. All gels were stained with methylene blue and destained with water.

\section{Generation of SUS1 constructs}

All SUS1 gene constructs contained the last $20 \mathrm{nt}$ of $5^{\prime} \mathrm{UTR}$. The I2 mutants (mut1, mut2, mut3, mut4, and mut1-I1 $\Delta$,) were constructed by the fusion PCR method (Yon and Fried 1989; Lesser and Guthrie 1993), using the primers specified in Supplemental Table S1. The constructs were cloned into a modified pRS425 vector containing the glyceraldehyde-3-phosphate dehydrogenase (GPD) promoter and the CUP1 gene as a reporter without ATG, followed by the first $200 \mathrm{nt}$ of the SUS1 gene $3^{\prime}$ UTR (see the scheme in Supplemental Fig. S5). Taq DNA polymerase (Roche) was used to amplify the WT, I2-mut1, I2-mut2, I2mut3, and I2-mut4 constructs of SUS1. Due to the presence of a high GC content in I2-mut3, the
Q5 Hot Start High-Fidelity DNA Polymerase (New England Biolabs) was used to obtain this construct.

\section{Yeast cultures and microbiological techniques}

Copper resistance assays were carried out by growing the transformed cup $1 \Delta$ cells at $30^{\circ} \mathrm{C}$ on synthetic selective medium (SC: glucose $2 \%$, ammonium sulphate $0.5 \%$, yeast nitrogen base $0.17 \%$, and supplements [Dropout]) lacking Leucine (Leu) to $0.4-0.5 \mathrm{OD}_{600}$. Subsequently, 10-fold serial dilutions of an equal number of cells were made and drops spotted onto SC-leu plates containing different concentration of $\mathrm{CuSO}_{4}$ (Lesser and Guthrie 1993). Plates were photographed after $3-5 \mathrm{~d}$ of incubation at $30^{\circ} \mathrm{C}$. Yeast cell transformations were done by the LiAc/SS carrier DNA/PEG method (Gietz and Schiestl 2007).

\section{RNA extraction, reverse transcription PCRs, and semi-q-RT-PCRs}

Total RNA was harvested from sus1 $\Delta$ cells transformed with the CUP1 plasmids bearing SUS1g, SUS1-I2-mut1, SUS1-I2-mut2, SUS1-I2-mut3, or SUS1-I2-mut4 by the Hot/Acid-phenol method (Schmitt et al. 1990), and quantified using a Nanodrop. RNA quality was checked by $1 \%$ agarose gels dyed with ethidium bromide $(\mathrm{EtBr})$. The cells were grown in $100 \mathrm{~mL}$ of SC-Leu at $30^{\circ} \mathrm{C}$ until $0.4 \mathrm{OD}_{600}$ and then divided into two equal aliquots, in order to incubate the cell cultures under two different conditions: the cells of the first aliquot were grown $2 \mathrm{~h}$ more at $30^{\circ} \mathrm{C}$ in SC-Leu and the cells of the second aliquot were collected by centrifugation, resuspended in equal volume of preheated $42^{\circ} \mathrm{C} \mathrm{SC}$-Leu media and incubated for $20 \mathrm{~m}$ at $42^{\circ} \mathrm{C}$. A $500 \mathrm{ng}$ of DNAse I-treated RNA was used to perform the reverse transcription PCR in each case. Reverse transcription was carried out using standard procedures, with random hexamers and M-MLV reverse transcriptase (Invitrogen). For semiquantitative RT-PCR, a specific pair of primers located upstream exon 1 and exon 3 was used to amplify the transcripts of SUS1 ( 25 cycles to quantify the mRNA and 35 cycles to amplify low abundant transcripts) and SCR1 (20 cycles). SCR1 levels are commonly used as loading control. The amplified products were run in a REALSAFE (Real laboratory) stained 3\% agarose gel and visualized with a BioRad UV CCD Camera. In all cases, negative controls that included all reagents except cDNA were included. The mRNA concentrations were normalized relative to $S C R 1$, and the accumulation of mRNA is represented relative to wild type. The mRNA bands were quantified by the ImageJ program (http://rsbweb.nih.gov/ij/)

\section{In situ hybridization (FISH)}

Fluorescent in situ hybridization (FISH) against poly(A) ${ }^{+}$RNA was done as described by Cuenca-Bono et al. (2011); yeast cells were grown in $100 \mathrm{~mL}$ of SC-Leu medium at $30^{\circ} \mathrm{C}$ to an $0.3 \mathrm{OD} 600$. Then, cultures were rapidly shifted to $37^{\circ} \mathrm{C}$ incubator for $2 \mathrm{~h}$. After hybridization, slides were mounted using VECTASHIELD Mounting Medium with DAPI. Detection of Cy3-oligo(dT) was performed using a Leica DM600B fluorescence microscope. mRNA accumulation was represented as the percentage of cells showing a bright signal in the nucleus that colocalized with DAPI staining (DNA). The percentages correspond to averages of at least three biological replicates. 


\section{Total protein extraction for histone modification detection and Western blot}

For the Western blot assays, the transformed sus $1 \Delta$ cells at $30^{\circ} \mathrm{C}$ were grown on $50 \mathrm{~mL}$ synthetic selective medium lacking Leucine (SCLeu) to $0.5 \mathrm{OD}_{600}$, then the cells were harvested and washed by $20 \%$ TCA, after nitrogen freezing the cells were thawed and resuspended in $0.5 \mathrm{~mL}$ of $20 \%$ TCA. Cells were disrupted by glass beads and the lysate was recovered. After centrifuging the pellet during $10 \mathrm{~min}(300 \mathrm{rpm})$ the pellet was resuspended in $200 \mu \mathrm{L}$ of $1 \times \mathrm{LB}$ plus $50 \mu \mathrm{L}$ of $2 \mathrm{M}$ unbuffered Tris and then boiled during $3 \mathrm{~min}$ at $95^{\circ} \mathrm{C}$. The suspension was centrifuged during $5 \mathrm{~min}$ at 3000 $\mathrm{rpm}$ and the supernatant was recovered. The proteins were separated by $15 \%$ SDS-PAGE and electrotransferred to nitrocellulose membranes, as previously described (Thiriet and Albert 1995). Membranes were stained with Ponceau S, as a transferring control, and were incubated with specific antibodies: $\alpha-\mathrm{H} 2 \mathrm{~B}$ ubiquitinated at position 123 (Cell Signaling) and a-PGK1 (Thermoscientific). Proteins were detected with horseradish peroxidase-conjugated anti-rabbit secondary antibodies and ECL Advanced reagents (GE Healthcare). Specific signals were quantified by the ImageJ program (http://rsbweb.nih.gov/ij/).

\section{SUPPLEMENTAL MATERIAL}

Supplemental material is available for this article.

\section{ACKNOWLEDGMENTS}

This work has been supported by Ministerio de Economía y Competitividad (MINECO) of Spain (BFU2011-23418 and BFU2014-57636-P to S.R.-N. and BFU-2012-30770 to J.G.), Generalitat Valenciana of Spain (PROMETEO/2013/061, ACOMP/ 2014/061, and ACOMP/2015/096 to S.R.-N., ACOMP/2014/056 to J.G., and a Santiago Grisolía fellowship to A.A.Q.), and Universidad Católica de Valencia. We are grateful to the undergraduate students Chiara Boccellato, Alejandro Aparicio, and Joan Serrano from the ERASMUS, ADEIT-UV, and SIE-UPV programs for making contributions to this work.

Received August 17, 2015; accepted October 3, 2015.

\section{REFERENCES}

Acuña LIG, Kornblihtt AR. 2014. Long range chromatin organization: a new layer in splicing regulation? Transcription 5: e28726.

Coolidge CJ, Seely RJ, Patton JG. 1997. Functional analysis of the polypyrimidine tract in pre-mRNA splicing. Nucleic Acids Res 25: 888896.

Cuenca-Bono B, García-Molinero V, Pascual-García P, Dopazo H, Llopis A, Vilardell J, Rodríguez-Navarro S. 2011. SUS1 introns are required for efficient mRNA nuclear export in yeast. Nucleic Acids Res 39: 8599-8611.

Darty K, Denise A, Ponty Y. 2009. VARNA: interactive drawing and editing of the RNA secondary structure. Bioinformatics 25: 1974-1975.

Deshler JO, Rossi JJ. 1991. Unexpected point mutations activate cryptic $3^{\prime}$ splice sites by perturbing a natural secondary structure within a yeast intron. Genes Dev 5: 1252-1263.

Dong S, Li C, Zenklusen D, Singer RH, Jacobson A, He F. 2007. YRA1 autoregulation requires nuclear export and cytoplasmic Edc3p-mediated degradation of its pre-mRNA. Mol Cell 25: 559-573.
Dong S, Jacobson A, He F. 2010. Degradation of YRA1 pre-mRNA in the cytoplasm requires translational repression, multiple modular intronic elements, Edc3p, and Mex67p. PLoS Biol 8: e1000360.

Fica SM, Tuttle N, Novak T, Li N-S, Lu J, Koodathingal P, Dai Q, Staley JP, Piccirilli JA. 2013. RNA catalyses nuclear pre-mRNA splicing. Nature 503: 229-234.

Fouser LA, Friesen JD. 1987. Effects on mRNA splicing of mutations in the $3^{\prime}$ region of the Saccharomyces cerevisiae actin intron. Mol Cell Biol 7: 225-230.

Gahura O, Hammann C, Valentová A, Půta F, Folk P. 2011. Secondary structure is required for $3^{\prime}$ splice site recognition in yeast. Nucleic Acids Res 39: 9759-9767.

Galan A, Rodríguez-Navarro S. 2012. Sus1/ENY2: a multitasking protein in eukaryotic gene expression. Crit Rev Biochem Mol Biol 47: 556-568.

García-Oliver E, García-Molinero V, Rodríguez-Navarro S. 2012. mRNA export and gene expression: the SAGA-TREX-2 connection. Biochim Biophys Acta 1819: 555-565.

Gietz RD, Schiestl RH. 2007. Quick and easy yeast transformation using the LiAc/SS carrier DNA/PEG method. Nat Protoc 2: 35-37.

González-Aguilera C, Tous C, Gómez-Gonzalez B, Huertas P, Luna R, Aguilera A. 2008. The THP1-SAC3-SUS1-CDC31 complex works in transcription elongation-mRNA export preventing RNA-mediated genome instability. Mol Biol Cell 19: 4310-4318.

Hossain MA, Claggett JM, Nguyen T, Johnson TL. 2009. The cap binding complex influences $\mathrm{H} 2 \mathrm{~B}$ ubiquitination by facilitating splicing of the SUS1 pre-mRNA. RNA 15: 1515-1527.

Hossain MA, Rodriguez CM, Johnson TL. 2011. Key features of the two-intron Saccharomyces cerevisiae gene SUS1 contribute to its alternative splicing. Nucleic Acids Res 39: 8612-8627.

Johnson TL, Vilardell J. 2012. Regulated pre-mRNA splicing: the ghostwriter of the eukaryotic genome. Biochim Biophys Acta 1819: 538-545.

Klöckner C, Schneider M, Lutz S, Jani D, Kressler D, Stewart M, Hurt E, Köhler A. 2009. Mutational uncoupling of the role of Sus1 in nuclear pore complex targeting of an mRNA export complex and histone H2B deubiquitination. J Biol Chem 284: 12049-12056.

Köhler A, Pascual-García P, Llopis A, Zapater M, Posas F, Hurt E, Rodríguez-Navarro S. 2006. The mRNA export factor Sus1 is involved in Spt/Ada/Gcn5 acetyltransferase-mediated H2B deubiquitinylation through its interaction with Ubp8 and Sgf11. Mol Biol Cell 17: 4228-4236.

Kopytova DV, Krasnov AN, Orlova AV, Gurskiy DY, Nabirochkina EN, Georgieva SG, Shidlovskii YV. 2010. ENY2: couple, triple...more? Cell Cycle 9: 479-481.

Kupfer DM, Drabenstot SD, Buchanan KL, Lai H, Zhu H, Dyer DW, Roe BA, Murphy JW. 2004. Introns and splicing elements of five diverse fungi. Eukaryot Cell 3: 1088-1100.

Kurshakova M, Maksimenko O, Golovnin A, Pulina M, Georgieva S, Georgiev P, Krasnov A. 2007. Evolutionarily conserved E(y)2/Sus1 protein is essential for the barrier activity of $\mathrm{Su}(\mathrm{Hw})$-dependent insulators in Drosophila. Mol Cell 27: 332-338.

Lesser CF, Guthrie C. 1993. Mutational analysis of pre-mRNA splicing in Saccharomyces cerevisiae using a sensitive new reporter gene, CUP1. Genetics 133: 851-863.

Liu X, Mertz JE. 1996. Sequence of the polypyrimidine tract of the $3^{\prime}$ terminal $3^{\prime}$ splicing signal can affect intron-dependent pre-mRNA processing in vivo. Nucleic Acids Res 24: 1765-1773.

Matlin AJ, Southby J, Gooding C, Smith CWJ. 2007. Repression of $\alpha-$ actinin SM exon splicing by assisted binding of PTB to the polypyrimidine tract. RNA 13: 1214-1223.

Meyer M, Plass M, Pérez-Valle J, Eyras E, Vilardell J. 2011. Deciphering $3^{\prime}$ ss selection in the yeast genome reveals an RNA thermosensor that mediates alternative splicing. Mol Cell 43: 1033-1039.

Moehle EA, Braberg H, Krogan NJ, Guthrie C. 2014. Adventures in time and space: splicing efficiency and RNA polymerase II elongation rate. RNA Biol 11: 313-319.

Pascual-García P, Govind CK, Queralt E, Cuenca-Bono B, Llopis A, Chavez S, Hinnebusch AG, Rodríguez-Navarro S. 2008. Sus1 is 


\section{AbuQattam et al.}

recruited to coding regions and functions during transcription elongation in association with SAGA and TREX2. Genes Dev 22: 2811-2822.

Pérez-Valle J, Vilardell J. 2012. Intronic features that determine the selection of the 3' splice site. Wiley Interdiscip Rev RNA 3: 707-717.

Preker P, Guthrie C. 2006. Autoregulation of the mRNA export factor Yralp requires inefficient splicing of its pre-mRNA. RNA 12: 9941006.

Rodríguez-Navarro S, Strässer K, Hurt E. 2002. An intron in the YRA1 gene is required to control Yral protein expression and mRNA export in yeast. EMBO Rep 3: 438-442.

Rodríguez-Navarro S, Fischer T, Luo M-J, Antúnez O, Brettschneider S, Lechner J, Pérez-Ortín JE, Reed R, Hurt E. 2004. Sus1, a functional component of the SAGA histone acetylase complex and the nuclear pore-associated mRNA export machinery. Cell 116: 75-86.

Rogic S, Montpetit B, Hoos HH, Mackworth AK, Ouellette BF, Hieter P. 2008. Correlation between the secondary structure of pre-mRNA introns and the efficiency of splicing in Saccharomyces cerevisiae. BMC Genomics 9: 355.

Roscigno RF, Weiner M, Garcia-Blanco MA. 1993. A mutational analysis of the polypyrimidine tract of introns. Effects of sequence differences in pyrimidine tracts on splicing. J Biol Chem 268: 1122211229.

Rymond BC, Rosbash M. 1985. Cleavage of $5^{\prime}$ splice site and lariat formation are independent of $3^{\prime}$ splice site in yeast mRNA splicing. Nature 317: 735-737.
Schmitt ME, Brown TA, Trumpower BL. 1990. A rapid and simple method for preparation of RNA from Saccharomyces cerevisiae. Nucleic Acids Res 18: 3091-3092.

Thiriet C, Albert P. 1995. Rapid and effective western blotting of histones from acid-urea-Triton and sodium dodecyl sulfate polyacrylamide gels: two different approaches depending on the subsequent qualitative or quantitative analysis. Electrophoresis 16: 357-361.

Wahl MC, Will CL, Luhrmann R. 2009. The spliceosome: design principles of a dynamic RNP machine. Cell 136: 701-718.

Wan Y, Qu K, Zhang QC, Flynn RA, Manor O, Ouyang Z, Zhang J, Spitale RC, Snyder MP, Segal E, et al. 2015. Landscape and variation of RNA secondary structure across the human transcriptome. Nature 505: 706-709.

Warf MB, Berglund JA. 2010. Role of RNA structure in regulating premRNA splicing. Trends Biochem Sci 35: 169-178.

Will CL, Lührmann R. 2011. Spliceosome structure and function. Cold Spring Harb Perspect Biol 3: a003707.

Yon J, Fried M. 1989. Precise gene fusion by PCR. Nucleic Acids Res 17: 4895.

Zhao Y, Lang G, Ito S, Bonnet J, Metzger E, Sawatsubashi S, Suzuki E, le Guezennec X, Stunnenberg HG, Krasnov A, et al. 2008. A TFTC/ STAGA module mediates histone $\mathrm{H} 2 \mathrm{~A}$ and $\mathrm{H} 2 \mathrm{~B}$ deubiquitination, coactivates nuclear receptors, and counteracts heterochromatin silencing. Mol Cell 29: 92-101.

Zuker M. 2003. Mfold web server for nucleic acid folding and hybridization prediction. Nucleic Acids Res 31: 3406-3415. 

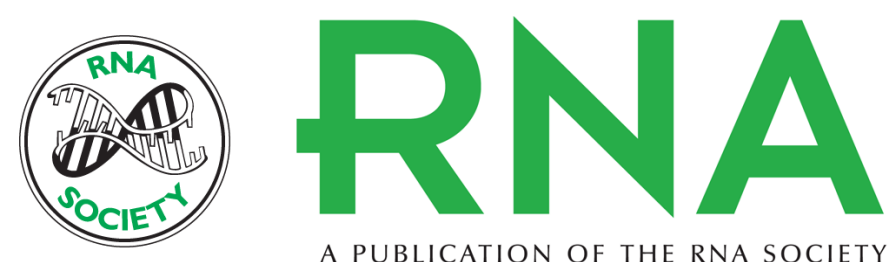

A PUBLICATION OF THE RNA SOCIETY

\section{An intronic RNA structure modulates expression of the mRNA biogenesis factor Sus1}

Ali AbuQattam, José Gallego and Susana Rodríguez-Navarro

RNA 2016 22: 75-86 originally published online November 6, 2015

Access the most recent version at doi:10.1261/rna.054049.115

\section{Supplemental http://rnajournal.cshlp.org/content/suppl/2015/11/03/rna.054049.115.DC1 \\ Material}

References This article cites 44 articles, 14 of which can be accessed free at: http://rnajournal.cshlp.org/content/22/1/75.full.html\#ref-list-1

Open Access Freely available online through the RNA Open Access option.

Creative This article, published in RNA, is available under a Creative Commons License Commons (Attribution 4.0 International), as described at

License http://creativecommons.org/licenses/by/4.0/.

Email Alerting Receive free email alerts when new articles cite this article - sign up in the box at the Service top right corner of the article or click here. 